\title{
Prominent pericardial and myocardial lesions in the Churg-Strauss syndrome (allergic granulomatosis and angiitis)
}

\author{
AG DAVISON, PJ THOMPSON, J DAVIES, B CORRIN, M TURNER-WARWICK \\ From the Brompton Hospital and Cardiothoracic Institute and the Hammersmith Hospital, London
}

The Churg-Strauss syndrome is a rare multisystem disease of unknown cause characterised by asthma, appreciable blood and tissue eosinophilia, angiitis, and necrotising granulomas. ${ }^{1}$ The clinical features are protean but cardiac lesions received little emphasis in the largest clinical series of patients so far reported. ${ }^{2}$ We report three patients with the Churg-Strauss syndrome who had prominent pericardial and myocardial lesions and also some previously undescribed clinical features.

\section{Case reports}

\section{CASE 1}

A 33 year old non-smoking woman, who had suffered for 11 years from mild asthma controlled with inhaled salbutamol, developed a painful rash, pericarditic chest pain, shortness of breath, left pleuritic pain, polymyalgia, and emotional lability. There was no family history of allergy. On examination she was febrile and had splinter haemorrhages, a raised, confluent, purplish rash on both arms, bilateral ankle oedema, and scattered wheezes.

When she was transferred to the Brompton Hospital, her rash was fading but she had developed weakness and anaesthesia in the distribution of her left popliteal and right radial nerves and the right dorsalis pedis, and both posterior tibial pulses were impalpable. The chest radiograph showed cardiomegaly, a left pleural effusion, and bilateral basal shadowing. The haemoglobin concentration was $9.0 \mathrm{~g} / \mathrm{dl}$, eosinophil count $15.2 \times 10 \% / 1$, and erythrocyte sedimentation rate (ESR) $39 \cdot \mathrm{mm}$ in the first hour. The serum IgE was $1300 \mathrm{IU} / \mathrm{ml}$ (normal 5-150 IU/ml) and circulating immune complexes containing $\operatorname{IgG}$ and IgA were detected. No autoantibodies or aspergillus precipitins were detected. Stool and serological tests for parasites gave negative results. Skin test responses to a standard range of 32 common environmental allergens were negative. Electrocardiographic and echocardiographic features were compatible with a pericardial effusion. An open pericardial biopsy showed infiltration by eosinophils, vasculitis, and giant cell granulomas. The pericardial fluid contained $7 \times 10^{9} / 1$ eosinophils.

The patient was treated initially with $60 \mathrm{mg}$ of prednisolone a day, the dose being reduced over four months to $20 \mathrm{mg}$ on alternate days. She was then symptom free, all pulses were palpable, and the neuropathy had almost totally resolved. She has remained well for over 18 months, maintained on alternate day prednisolone, with no haematological or immunological abnormalities.

Address for reprint requests: Dr Anthony G Davison, Brompton Hospital, London SW3 6HP.

Accepted 16 May 1983
CASE 2

A 32 year old man presented with a history of anorexia, weight loss, fever, intermittent joint pains, and continuous retrosternal chest pain. At 17 years of age he had developed asthma; for the last two years his only treatment had been choline theophyllinate and inhaled beclomethasone dipropionate.

On examination, the pulse was 120 beats/min, blood pressure $90 / 60 \mathrm{~mm} \mathrm{Hg}$, and jugular venous pressure raised $6 \mathrm{~cm}$; and there was a gallop rhythm. There were bilateral wheezes and crackles in the lungs. The chest radiograph showed cardiomegaly. The haemoglobin concentration was $11.7 \mathrm{~g} / \mathrm{dl}$, white cell count $11.4 \times 10 \% / 1$, and absolute eosinophil count $4.7 \times 10 \%$. The ESR was $104 \mathrm{~mm}$ in the first hour. Stool and serological tests for parasites gave negative results. Autoantibodies and aspergillus precipitins were not detected. Serum IgE was normal. Skin test responses to a standard range of 32 common environmental allergens were negative. Tests of renal function gave normal results. Liver enzyme levels were raised and a liver biopsy showed a focal non-necrotising granuloma with occasional eosinophils.

Electrocardiographic and echocardiographic features were compatible with a pericardial effusion. Right heart catheterisation showed normal pressure (including pulmonary artery wedge pressure) and an angiogram showed normal cardiac function. Open pericardial biopsy showed that the pericardium was thickened by fibrosis and densely infiltrated with eosinophils, and contained prominent necrotising epithelioid and giant cell granulomas. The pericardial fluid was sterile and contained no eosinophils. The patient improved with diuretic treatment but still had a raised ESR and blood eosinophil count on discharge.

Two months later he was readmitted in cardiac failure. His full blood count and ESR were now normal. Electrocardiograms showed left axis deviation and poor $\mathbf{R}$ wave progression. A chest radiograph showed cardiomegaly with upper lobe blood diversion and bilateral pleural effusions. Pericardial effusion was not indicated by echocardiography. Cardiac catheterisation showed a raised right ventricular pressure of $55 / 18 \mathrm{~mm} \mathrm{Hg}$ and a raised left ventricular end diastolic pressure of $22 \mathrm{~mm} \mathrm{Hg}$. Pressure tracings did not suggest restrictive heart disease. Angiograms showed dilated and poorly functioning ventricles with mild incompetence of both mitral and tricuspid vales. There was no evidence of fibrosis or thrombosis. Right ventricular endomyocardial biopsy specimens were normal. The patient was given anticoagulant treatment and increased doses of diuretics and vasodilators with good effect.

A year later he was admitted with severe asthma. There was no eosinophilia; serum IgE was $280 \mathrm{IU} / \mathrm{ml}$ (normal 
5-150 IU $/ \mathrm{ml}$ ). The asthma improved with prednisolone and bronchodilator treatment. He was maintained on these drugs in addition to his previous treatment, and remained well for 18 months. He then developed a rash while taking prednisolone $15 \mathrm{mg} /$ day. Circulating immune complexes containing complement fraction $\mathrm{Clq}$, IgG, and IgA were detected. Skin biopsy showed a small vessel angiitis with considerable lymphocyte and neutrophil polymorphonuclear infiltration. The rash resolved after the addition of azathioprine (100 $\mathrm{mg} /$ day) to the medication.

CASE 3

A woman developed hay fever, nasal polyps, and asthma (non-atopic) in 1972 when 35 years of age. Later that year she developed widespread bilateral shadows on the chest radiograph and had an eosinophil count of $9.5 \times 10 \% 1$, haemoglobin concentration of $9.9 \mathrm{~g} / \mathrm{dl}$, and ESR of $112 \mathrm{~mm}$ in the first hour, for which no cause was found. There was no family history of atopy. The patient was treated with a course of hydrocortisone and improved.

Similar episodes occurred in 1973, 1974, 1975, 1976, and 1979. Additional features noted at these times included joint pains, pericarditis, epigastric pain, cervical lymphadenitis, paraesthesiae down the outer aspect of the left arm and a serum IgE concentration of $840 \mathrm{IU} / \mathrm{ml}$. Prednisolone produced clinical improvement on each occasion.

In February 1981, while taking prednisolone $4 \mathrm{mg} /$ day, she developed a tender submandibular swelling, further lung shadows, and eosinophilia. In June 1981 she transferred to the Brompton Hospital after the development of pleuritis and pericarditis. Splinter haemorrhages were present under the nails and the left posterior tibial and the right dorsalis pedis pulses were absent. Renal and hepatic function tests were normal. No autoantibodies or aspergillus precipitins were detected. Circulating immune complexes containing Clq, IgM, and IgA were found. The electrocardiographic and echocardiographic findings were consistent with a pericardial effusion. A pleural biopsy showed eosinophilic infiltration and granulomas.

The dose of prednisolone was increased and after two months the patient was symptom free, all her pulses were palpable, the pericardial and pleural effusions had resolved, and the ESR and the eosinophil count were normal. She has remained well on alternate day prednisolone.

\section{Discussion}

In the original description of allergic granulomatosis and angiitis, ${ }^{1}$ the heart was frequently found to be affected at necropsy, but there was little reference to myocardial disease and none to pericardial disease in the clinical series of 30 patients reported by Chumbley et al. ${ }^{2}$ Similarly, the 1980 WHO/ISFC Task Force on the definition of cardiomyopathies did not include allergic granulomatosis as a cause of heart muscle disease. ${ }^{3}$ All of our patients had pericardiäl disease and the second patient also had myocardial disease. The latter resulted in cardiac dilatation and had to be distinguished from idiopathic congestive cardiomyopathy ${ }^{3}$ and idiopathic hypereosinophilic endomyocardial disease. ${ }^{4}$ The former lacks the eosinophilia, asthma, and vasculitis of the Churg-Strauss syndrome, whereas in the latter there is characteristically a restrictive $\mathscr{S}$ cardiac defect associated with a prolonged eosinophilia of unknown cause.

In addition to myocardial and pericardial disease, our $\bar{\omega}$ patients also had hepatic, pulmonary, pleural, lymph node, $\overparen{\mathbb{D}}$ peripheral nerve, muscle, joint, and skin lesions and $\frac{2}{2}$ anaemia, thus illustrating the multisystem disease which may occur in the Churg-Strauss syndrome. The subman- $\overrightarrow{0}$ dibular gland enlargement and transient vascular lesions :with loss of peripheral pulses that occurred in two of our $\vec{\omega}$ patients do not appear to have been reported previously in this disease. The emotional lability of our first patient, which improved after treatment, may perhaps reflect additional central nervous system disease.

The major pathological lesions of the Churg-Strauss $\overrightarrow{0}$ syndrome may not be present together and a single biopsy may fail to show all the characteristic features.' By itself, $\omega$ an arteritis is not diagnostic, but necrotising granulomas with intense eosinophilic infiltration in a patient with asthma and blood eosinophilia provide strong evidence of the Churg-Strauss syndrome and distinguish the condition from polyarteritis nodosa.

Asthma and eosinophils suggest allergy, but the atopic $\stackrel{\mathbb{D}}{\square}$ state of patients with Churg-Strauss syndrome has seldom $\vec{\theta}$ been clarified and no specific allergen has been identified. $\mathscr{\oplus}$ None of our cases had positive immediate responses to $\omega$ hypersensitivity tests or a family history of allergy. Raised serum IgE concentrations have been reported ${ }^{2} \mathrm{~s}$ and in two of our patients the level was raised when the disease was active and fell with treatment, but in our second case the reverse occurred. The extent of increase in IgE was moderate by comparison with the very high blood eosino- $\varrho$ phil count. The clinical relevance of the high IgE concen- $\overrightarrow{\overrightarrow{0}}$ trations is uncertain. Circulating immune complexes were $\frac{3}{3}$ detected and eosinophils may have a role in immune complex clearance; ${ }^{6}$ immune complexes have, however, been detected in other types of vasculitis. ${ }^{7}$

A diagnosis of Churg-Strauss syndrome should be considered in any asthmatic patient who develops evidence of cardiac disease (or other multisystem disease) and a high ESR or a high eosinophil count. Specific treatment is required and will differ from treatment for asthma with unrelated cardiac disease-for example, idiopathic 0 cardiomyopathy. Favourable responses to corticosteroids, ${ }^{2} \supsetneq$ azathioprine, ${ }^{2}$ combinations of corticosteroid and $\frac{\text { ? }}{2}$ azathioprine, ${ }^{8}$ and cyclophosphamide ${ }^{7}$ have been described in the Churg-Strauss syndrome and a five year survival of $62 \%$ after the start of treatment has been reported. ${ }^{2}$ Continuous treatment is usually required to prevent relapse. $\mathrm{N}$

We thank Professor JF Goodwin and Dr CJF Spry for their help, and Miss H Rolls and Miss T Chudleigh for typing the $\omega$ manuscript.

\section{References}

1 Churg J, Strauss L. Allergic granulomatosis, allergic angiitis and periarteritis nodosa. Am J Pathol 1951;27:227-310.

2 Chumbley LC, Harrison EG, De Remee RA. Allergic granulomatosis and angiitis (Churg-Strauss Syndrome). Mayo $\cong$ Clin Proc 1977;52:477-81. 
${ }^{3}$ Report of the WHO/ISFC Task Force on the Definition and Classification of Cardiomyopathies. Br Heart J 1980;44:6723.

${ }^{4}$ Chew CYC, Ziady GM, Raphael MJ, Nellen M, Oakley CM Primary restrictive myopathy. Non-tropical endomyocardial fibrosis and hypereosinophilic heart disease. $\mathrm{Br}$ Heart $\mathrm{J}$ 1977;39:399-412.

5 Koss MN, Antonovych T, Hochholzer L. Allergic granulomatosis (Churg-Strauss Syndrome). Am J Surg Pathol 1981;5:21-8.
'Fauci AS. The idiopathic hypereosinophilic syndrome. Ann Intern Med 1982;97:78-92.

' Fauci AS, Haynes BF, Katz P. The spectrum of vasculitis. Ann Intern Med 1978;89:660-76.

${ }^{8}$ Cooper BJ, Bacal E, Patterson R. Allergic angiitis and granulomatosis. Prolonged remission induced by combined prednisolone-azathioprine therapy. Arch Intern Med 1978;138:367-71. 